\title{
Time-frequency analysis of band-limited EEG with BMFLC and Kalman filter for $\mathrm{BCI}$ applications
}

\author{
Yubo Wang, Kalyana C Veluvolu* and Minho Lee
}

\begin{abstract}
Background: Time-Frequency analysis of electroencephalogram (EEG) during different mental tasks received significant attention. As EEG is non-stationary, time-frequency analysis is essential to analyze brain states during different mental tasks. Further, the time-frequency information of EEG signal can be used as a feature for classification in brain-computer interface $(\mathrm{BCl})$ applications.

Methods: To accurately model the EEG, band-limited multiple Fourier linear combiner (BMFLC), a linear combination of truncated multiple Fourier series models is employed. A state-space model for BMFLC in combination with Kalman filter/smoother is developed to obtain accurate adaptive estimation. By virtue of construction, BMFLC with Kalman filter/smoother provides accurate time-frequency decomposition of the bandlimited signal.

Results: The proposed method is computationally fast and is suitable for real-time $\mathrm{BCl}$ applications. To evaluate the proposed algorithm, a comparison with short-time Fourier transform (STFT) and continuous wavelet transform (CWT) for both synthesized and real EEG data is performed in this paper. The proposed method is applied to $\mathrm{BCl}$ Competition data IV for ERD detection in comparison with existing methods.
\end{abstract}

Conclusions: Results show that the proposed algorithm can provide optimal time-frequency resolution as compared to STFT and CWT. For ERD detection, BMFLC-KF outperforms STFT and BMFLC-KS in real-time applicability with low computational requirement.

Keywords: Time-Frequency analysis, Band limited multiple fourier linear combiner, Adaptive filter, Kalman filter, Smoother, EEG

\section{Introduction}

EEG uses electrodes to record electrical brain activity that originates from the post-synaptic potentials, aggregates at the cortex, and transfers through the skull to the scalp. The EEG signal is the reflection of brains neuronal oscillations. These oscillations with similar frequency and energy lead to the separation of frequency bands [1]. Numerous studies have tried to identify the relation between the frequency bands and brain states and it still remains as a hotspot of ongoing neuroscience research $[2,3]$. EEG activity related with voluntary movements has been the center of interest, as it is applicable to brain-computer interfaces (BCI) [4-8].

*Correspondence: veluvolu@ee.knu.ac.kr

College of IT Engineering, Kyungpook National University, 1370 Sanyuk-dong, Daegu, 702-701, South Korea
Several BCI systems rely on an amplitude attenuation phenomenon, namely event-related desynchronization (ERD) that can be voluntarily controlled by movement imagery. It was shown in [9] that during both planning and execution of hand movements, the ERD can be detected in most of the subjects within the band of $\mu$-rhythm $(6-14 \mathrm{~Hz})$. By utilizing this amplitude attenuation phenomenon, an alternative communication pathway can be built directly from human brain to the computer [4]. The accuracy of this class of methods was examined in [6]. In recent years, this type of BCIs has been applied for limb function recovery [10] and robotic system control [7], which can improve the quality of life of the subject with severe motor function impairment. The energy decrease in ERD usually occurs in a specific frequency band for a subject. When the frequency characteristics of signal 
are required, the fast Fourier transform (FFT) is often used. For BCI applications, the ERD in the EEG signal is considered as a percentage change of the signal amplitude with respect to a experiment cue [9,11-13]. Since the FFT-based methods cannot provide time-frequency information, the time-frequency representation (TFR) of EEG signal is extremely important for ERD analysis.

As the performance of EEG-based BCI systems rely on the time-domain and frequency-domain features, a variety of EEG features (such as power spectrum within a predefined frequency band [14] and phase lock value [15]) that reflects ongoing brain states have been attempted for designing BCI systems. In general, the feature extraction algorithm requires accumulation of sufficient number of samples for generating control commands. In [16], an EEG-based BCI for three-dimensional movement control has been implemented where the power spectrum was calculated for every $50 \mathrm{~ms}$ with a 16-order autoregressive(AR) model. In a recent research [17], BCI with a noninvasive functional electrical stimulation has been studied. In the on-line processing phase, the $\mathrm{BCI}$ aggregates $500 \mathrm{~ms}$ EEG signal and then FFT is applied to obtain power estimates for classification. It is clear that the response time of BCIs mainly depends on the time required by the feature extraction algorithm to store and process a sufficient number of samples. Since power spectrum is a frequency domain feature, the time-frequency representation(TFR) methods that can provide amplitude variation along time axis, can be directly applied to BCI systems. Furthermore, the accuracy of BCIs can be improved by employing a narrow subject-specific frequency band $[9,18,19]$. In [18], an adaptive filtering approach was employed for identifying the subjectspecific frequency band to improve the classification accuracy.

The TFR methods can be categorized into two types, namely non-parametric and parametric methods. The non-parametric TFRs such as band-pass filtering, short time Fourier transform (STFT) and continuous wavelet transform (CWT) were successfully applied in timefrequency analysis of EEG [20,21]. However, all the traditional methods have pros and cons in temporal and spectral resolutions. In band-pass filtering, the temporal and spectral resolution is highly dependent on the filter type, center frequency of the filter and its order. The temporal and spectral resolution of STFT is determined by the window length. The CWT can be considered as the best TFR technique among the available methods. However, it still suffers with the tradeoff between temporal and spectral resolution as STFT. The computational requirement of CWT remains as a major barrier for real-time BCI applications. A performance comparison of all the TFR methods for EEG time-frequency analysis can be found in [22].
Recently, a new method band-limited multiple Fourier linear combiner (BMFLC) was developed for estimating band-limited signals within a pre-defined frequency band [18]. By incorporating the idea of linear time varying model with a fixed frequency band, BMFLC can provide an alternative spectral estimation method for band-limited signals. The original BMFLC adopted a truncated Fourier series as the model and estimated the Fourier coefficients by least mean squares (LMS) algorithm $[23,24]$. As LMS requires time to converge to the steady-state, the algorithm accuracy cannot be guaranteed for small data segments. Especially, LMS algorithm is not suitable when the main objective is to accurately track the amplitude changes of a band-limited signal. To improve the accuracy of the time-frequency decomposition and the tracking ability of the existing BMFLC for real-time applications, Kalman Filter is employed. The proposed method is designed to extract time-frequency amplitude distribution that is more suitable for $\mathrm{BCI}$ applications. The performance of proposed method is evaluated in comparison with STFT, CWT and the existing BMFLC-LMS method.

\section{Methods}

This section first reviews the existing methods for timefrequency representation and later presents the proposed methods.

\section{Classical time-frequency methods}

As the traditional fast Fourier transform (FFT) does not provide time-domain information, the intuitive way to overcome this is to isolate the signal in time domain by multiplying with a window function and compute the Fourier coefficients in that time interval, then shift the time window through the time line to capture the entire time-frequency information of the signal [25].

In [26], the time-frequency resolution of STFT is interpreted by the Heisenberg uncertainty principle. It asserts that the temporal and spectral resolutions cannot be guaranteed at the same time and the joint time-frequency resolution has a lower bound given by

$$
\triangle t^{2} \triangle \omega^{2}=\frac{1}{2}
$$

It can be illustrated as a box centered at $(t, \omega)$, with the length equal to $\Delta t$ in time domain and the width equal to $\Delta \omega$ in the frequency domain, to sweep the whole timefrequency domain to extract the time-frequency information. Since this product remains constant, the increase of one quantity will cause degradation in the other. This degradation (leakage effect) is also due to this constant product. Thus the information that can be extracted via STFT is actually the information within that box. If the window function has a Gaussian envelope, the STFT can 
achieve the lower bound defined in (1) [22]. The STFT with a Gaussian window function is commonly referred as Gabor transform.

In STFT, after fixing the length of the window function, its time-frequency resolution remains constant for the entire time-frequency domain. The continuous wavelet transform (CWT) solves this problem by adopting a dilated and translated versions of the same function namely, the mother wavelet [26,27]. The dilated version of the mother wavelet is controlled by a scalar parameter $a$ in CWT, where the corresponding time-frequency resolution can be provided as $\frac{\Delta t}{a} \times a \triangle \omega=\frac{1}{2}$. Although the time-frequency resolution is still bounded by Heisenberg uncertainty, but the length and width are scaled by the parameter $a$. Therefore, for CWT the time-frequency resolution can be adjusted by the parameter $a$ compared to a fixed time-frequency resolution in STFT.

If the mother wavelet is a complex function, then the CWT is also known as complex CWT which is widely used in EEG signal processing [12,22,28]. Similar to STFT, the joint time-frequency resolution is optimized by a mother wavelet that has Gaussian envelope [22]. Morlet wavelet is one of them and is defined as [29]:

$$
\psi_{\text {Morlet }}(t)=\pi^{-1 / 4} \cdot\left(e^{j \omega_{0} t}-e^{-\omega_{0}^{2} / 2}\right) \cdot e^{-t^{2} / 2}
$$

where $\omega_{0}$ defines the oscillation of the mother wavelet. The $e^{-\omega_{0}^{2} / 2}$ term in (2) is a correction term employed to satisfy the admissibility condition. If a sufficiently large $\omega_{0}$ is adopted, e.g. $\omega_{0}>5$, then the term can be neglected. The joint time-frequency resolution can achieve its lower bound with $\Delta t=0.707$ and $\triangle \omega=0.707$ [30]. For Morlet wavelets that have a dominant periodic component, the relationship between scale and Fourier frequency [30] can be obtained as

$$
f=\frac{\omega_{0}}{2 \pi a}
$$

where $f$ and $a$ represent the Fourier frequency and wavelets scale respectively.

The STFT and CWT belong to the analytic timefrequency representation methods, where the timefrequency resolution has a lower bound that is determined by the Heisenberg uncertainty. To apply the CWT and STFT to a signal, an appropriate length of the signal is required, as all the samples within the window function should be used at each time-frequency decomposition. The power spectrum defined in STFT and CWT is relative to the true signal spectrum that can be obtained from FFT. For some applications where the exact measurement of amplitude or power at a specific frequency is required, the CWT and STFT are not suitable.

\section{Band-limited multiple Fourier linear combiner}

The Fourier linear combiner (FLC) proposed in [31] works by adaptively estimating the Fourier coefficients of a known base frequency together with its harmonics with the help of the least mean squares (LMS) algorithm. In $[18,23]$, to estimate the unknown bandlimited signal, a pre-defined frequency band $\left[\omega_{1}-\omega_{n}\right]$ is considered and divided into ' $n$ ' finite number of divisions. Then n-FLC's are combined to form the BMFLC to estimate bandlimited signals. The frequency resolution of BMFLC, (i.e. $\triangle f=\frac{\Delta \omega}{2 \pi}$ ), is the frequency gap between two adjacent frequency components. The selection of frequency gap is a balance between signal characteristics and analysis requirement.

The signal model adopted by BMFLC $[18,32]$ is given by

$$
y_{k}=\sum_{r=1}^{n} a_{r k} \sin \left(\omega_{r} k\right)+b_{r k} \cos \left(\omega_{r} k\right)
$$

where $y_{k}$ denotes the estimated signal at sampling instant $k . a_{r k}$ and $b_{r k}$ represents the adaptive weights corresponding to the frequency $\omega_{r}$ at time instant $k$. The frequency components $\mathbf{x}_{k}$ and the corresponding adaptive weights $\mathbf{w}_{k}$ can be written in the matrix form as:

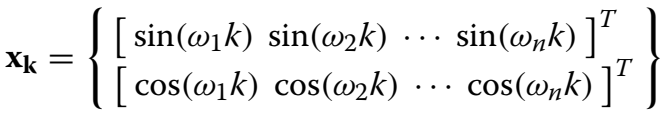

$$
\begin{aligned}
& \mathbf{w}_{k}=\left\{\begin{array}{l}
{\left[\begin{array}{llll}
a_{1 k} & a_{2 k} & \cdots & a_{n k}
\end{array}\right]^{T}} \\
{\left[\begin{array}{cccc}
b_{1 k} & b_{2 k} & \cdots & b_{n k}
\end{array}\right]^{T}}
\end{array}\right\}
\end{aligned}
$$

By employing LMS algorithm [33], the estimation of Fourier coefficients $\hat{\mathbf{w}}_{k}$ can be achieved by computing the following recursive equation:

$$
\begin{array}{r}
\epsilon_{k}=y_{k}-\mathbf{x}_{k}^{T} \hat{\mathbf{w}}_{k} \\
\hat{\mathbf{w}}_{k+1}=\hat{\mathbf{w}}_{k}+2 \mu \mathbf{x}_{k} \epsilon_{k}
\end{array}
$$

where $\epsilon_{k}$ is the error between modeling and measurement and $\mu$ is adaptive gain of LMS. As LMS algorithm relies on gradient based method for error minimization, the accuracy of the algorithm can be affected by the dynamic changes in the characteristics of the signal. LMS algorithm has only a single adjustable parameter for controlling the convergence rate, namely, the adaptive gain $\mu$. Selection of proper $\mu$ is very important for stability and convergence of the algorithm. For difference choices of frequency gap $(\triangle f)$, different values of $\mu$ are required to ensure stability and convergence [18]. As shown in [18], BMFLC-LMS requires around 5 seconds for the initial weights to track the amplitude changes in a $25 \mathrm{~s}$ EEG signal. For the practical BCI systems, the length of EEG signal is generally in a few seconds after the experiment cue. To improve the performance, we employ Kalman Filter (KF). 


\section{BMFLC with Kalman filter (BMFLC-KF) for real-time estimation}

The BMFLC signal model can be rewritten in the condensed form as

$$
y_{k}=\mathbf{x}_{k}^{T} \mathbf{w}_{k}+v_{k}
$$

where $\mathbf{x}_{k}$ and $\mathbf{w}_{k}$ are defined in (5) and (6). Clearly, this is a linear model with $\mathbf{x}_{k}$ being the frequency component at each time step $k$ and $v_{k}$ the observation error. Together with (5) and (6), and the output Equation (9), we have the state-space form for the BMFLC. The architecture of the proposed algorithm is illustrated in Figure 1.

The adaptive vector $\mathbf{w}_{k}$ in this model is considered to be state vector. The variation of the state when no priori information is available is typically described with the random walk model [34]. The state equation can now expressed as

$$
\mathbf{w}_{k+1}=\mathbf{w}_{k}+\eta_{k}
$$

where $\eta_{k}$ is the state error.

We assume that the measurement error $v_{k}$ and state error $\eta_{k}$ are uncorrelated, zero mean, Gaussian white noise processes and are denoted as

$$
\begin{gathered}
v \sim N(0, R) \\
\eta \sim N(0, \mathbf{Q})
\end{gathered}
$$

where $R$ and $\mathbf{Q}$ are measurement error covariance and state error covariance respectively. By employing the Kalman filter, the optimal estimation of the state of the dynamical system at time instant $k$ can be obtained with the measurement sequence $Y_{1: k-1}=\left[y_{1}, y_{2}, \cdots, y_{k-1}\right]$ where $y_{k}$ is the output defined in (4). Although the premise of the noise may not hold for the signal to be analyzed, it was shown in $[35,36]$ that Kalman filter can also provide the minimum mean-squared error estimation within the class of linear estimators. The adaptive algorithm together with the BMFLC is shown in Figure 1.

Throughout this section, we employ the the following notation:

$$
\hat{\mathbf{w}}_{k}=\mathbf{E}\left[\mathbf{w}_{k} \mid \mathbf{y}_{k-1}\right]
$$

where $\mathbf{E}\left[\mathbf{w}_{k} \mid \mathbf{y}_{k-1}\right]$ denotes the mathematical expectation of $\mathbf{w}$ at time instant $k$ with respect to previous observation $\mathbf{y}$ at $k-1$. Given the measurement sequence $Y_{1: k-1}$, the estimated state $\left(\hat{\mathbf{w}}_{k}\right.$ along with the estimated state error covariance $\mathbf{P}_{k}$ ) can be obtained with the Kalman filter as:

$$
\begin{aligned}
& \mathbf{K}_{k}=\mathbf{P}_{k} \mathbf{x}_{k}^{T}\left[\mathbf{x}_{k}^{T} \mathbf{P}_{k} \mathbf{x}_{k}+R\right]^{-1} \\
& \hat{\mathbf{w}}_{k+1}=\hat{\mathbf{w}}_{k}+\mathbf{K}_{k}\left(y_{k}-\mathbf{x}_{k}^{T} \hat{\mathbf{w}}_{k}\right) \\
& \mathbf{P}_{k+1}=\left[\mathbf{I}-\mathbf{K}_{k} \mathbf{x}_{k}\right] \mathbf{P}_{k}+\mathbf{Q}
\end{aligned}
$$

with initial condition $\hat{\mathbf{w}}_{0}, \mathbf{P}_{0} . \mathbf{K}_{k}$ is the Kalman gain updated at each time instant. The BMFLC-KF does not require the matrix inverse as it only involves a scalar observation. The proposed BMFLC-KF is computationally fast and is well suited for real-time applications.

\section{BMFLC with Kalman Smoother (BMFLC-KS) for off-line analysis}

Furthermore, if the future measurements $Y_{k+1: N}$ are available, they can be used to improve the accuracy of the state estimation. Hence the estimator is named as a smoother. In this paper, we adopt a fixed-interval smoother to improve the state estimation accuracy. The fixed-interval smoothing problem is to find the minimum mean square estimator $\hat{\mathbf{w}}_{k}$ for each state $\mathbf{w}_{k}(k=1, \cdots, N)$ given the observations $y_{1}, \cdots, y_{N}$.

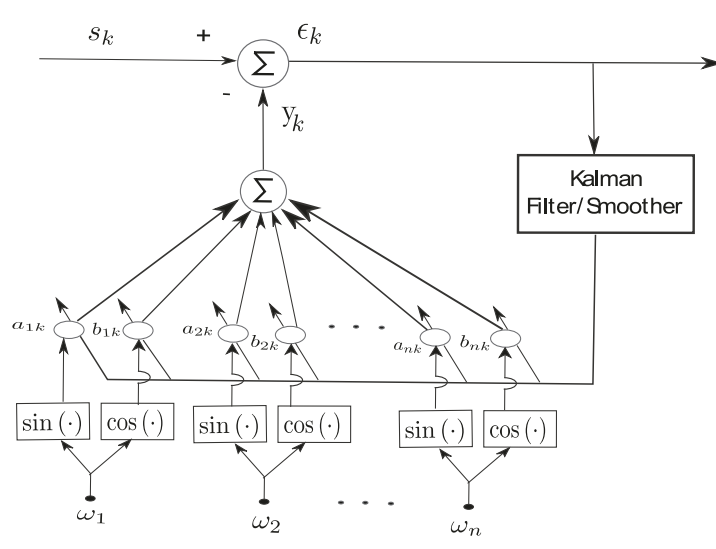

(a)

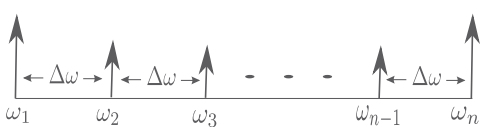

(b)

Figure 1 Diagram of BMFLC. (a) BMFLC Architecture; (b) Frequency components distribution. 
The smoothed estimator denoted by $\mathbf{w}_{k}^{N}$ can be obtained as follows [35]:

$$
\begin{aligned}
& \mathbf{w}_{k-1}^{N}=\mathbf{w}_{k-1}^{k-1}+\mathbf{J}_{k-1}\left(\mathbf{w}_{k}^{N}-\mathbf{w}_{k}^{k-1}\right) \\
& \mathbf{P}_{k-1}^{N}=\mathbf{P}_{k-1}^{k-1}+\mathbf{J}_{k-1}\left(\mathbf{P}_{k}^{N}-\mathbf{P}_{k}^{k-1}\right) \mathbf{J}_{k-1}^{T} \\
& \mathbf{J}_{k-1}=\mathbf{P}_{k-1}^{k-1}\left[\mathbf{P}_{k}^{k-1}\right]^{-1}
\end{aligned}
$$

where $\mathbf{w}_{k}^{s}=\mathbf{E}\left[\mathbf{w}_{k} \mid \mathbf{y}_{s}\right]$ and $\mathbf{P}_{k}^{s}=\mathbf{E}\left[\left(\mathbf{w}_{k}-\mathbf{w}_{k}^{s}\right)\left(\mathbf{w}_{k}-\mathbf{w}_{k}^{s}\right)^{T}\right]$ are estimated through recursion with the Kalman filter. The smoother estimation is then obtained by running the stored estimates backward in time. This procedure is suitable for off-line analysis.

The convergence properties of the proposed method are determined by the Kalman filter. The convergence analysis for autoregressive(AR) model with Kalman filter was well documented in [37-40]. It was shown in [39] that the Kalman filter is uniformly exponentially stable, if the output matrix sequence and the covariance of state error are bounded. In proposed method, the output matrix $\mathbf{x}_{k}^{T}$ in (9) is a combination of user-defined frequency components. As sine and cosine functions are bounded, the output matrix $\mathbf{x}_{k}^{T}$ is bounded. The covariance of state error is user-defined and is bounded. Hence the convergence of proposed algorithm can be established similar to [39]. Further the convergence rate can be quantified as in [40].

\section{Time-frequency decomposition with BMFLC}

Based on (9) and (10), the weight vectors of BMFLC represents the Fourier coefficients of the band-limited signal. We can combine the weights into the following form:

$$
\mathbf{w}_{k}^{f}=\left[\sqrt{a_{1 k}^{2}+b_{1 k}^{2}} \cdots \sqrt{a_{n k}^{2}+b_{n k}^{2}}\right]^{T}
$$

where $\mathbf{w}_{k}^{f}$ is the absolute weight vector of the frequency components at time instant $k$. The absolute timefrequency weights decomposed matrix $\mathbf{D}$ can be obtained for the signal with $m$ samples as

$$
\mathbf{D}=\left[\mathbf{w}_{1}^{f} \cdots \mathbf{w}_{k}^{f} \cdots \mathbf{w}_{m}^{f}\right]=\left[\begin{array}{ccc}
\sqrt{a_{11}^{2}+b_{11}^{2}} & \cdots & \sqrt{a_{1 m}^{2}+b_{1 m}^{2}} \\
\sqrt{a_{21}^{2}+b_{21}^{2}} & \cdots & \sqrt{a_{2 m}^{2}+b_{2 m}^{2}} \\
\vdots & \ddots & \vdots \\
\sqrt{a_{n 1}^{2}+b_{n 1}^{2}} & \cdots & \sqrt{a_{n m}^{2}+b_{n m}^{2}}
\end{array}\right]
$$

where each row vector presents the amplitude variation of a single frequency component at each time instant $k$. The energy distribution in the time-frequency mapping can be obtained as

$$
\text { Power }=\mathbf{D} \odot \mathbf{D}
$$

where the operator $\odot$ represents the element by element multiplication of the matrix. The $\mathbf{D}$ matrix provide the time-domain information of all individual frequency component and it can be directly used for time-frequency representation.

Comparing with the LMS based BMFLC in [18,32], by adopting the Kalman filter combined with the smoother procedure, an accurate weights adaption process in BMFLC can be achieved. Hence it provides an accurate time-frequency decomposition. The usage of smoother is optional and it depends on the purpose of analysis. For the off-line analysis, if an accurate time-frequency mapping is required, the smoother procedure can be employed.

\section{Data sets}

In order to compare the temporal and spectral resolutions, three synthesized signals are used in this study. Since EEG $\mu$-band $(6-14) \mathrm{Hz}$ is of special interest, three synthesized signals are chosen to contain the frequency components within this frequency band. The first synthesized signal is defined as:

$$
S_{1}(t)= \begin{cases}4 \sin (2 \pi 9 t)+2 \sin (2 \pi 11 t) ; & 0 \leq t<5 \\ 2 \sin (2 \pi 7 t)+4 \sin (2 \pi 14 t) ; & 5 \leq t \leq 10\end{cases}
$$

and is illustrated in Figure 2(a1). The signal has discontinuities in frequencies and has both amplitude and frequency modulations. This signal is used to test the frequency resolution and tracking ability of the algorithm. The second synthesized signal is defined as

$S_{2}(t)=\left\{\begin{array}{rc}4 \sin (2 \pi 10 t)+2 \sin (2 \pi 9 t) ; & 0 \leq t \leq 5,7 \leq t \leq 12,14 \leq t \leq 20 \\ 0 ; & \text { otherwise }\end{array}\right.$

and is shown in Figure 2(b1). The sudden burst in the signal is well-suited to test the temporal resolution of the method. To further analyze the spectral resolution capabilities of all the methods, a signal with four closely spaced frequency components is chosen as

$$
\begin{aligned}
S_{3}(t)= & 4 \sin (2 \pi 8.2 t)+3 \sin (2 \pi 8.6 t)+2 \sin (2 \pi 9 t) \\
& +4 \sin (2 \pi 9.6 t)
\end{aligned}
$$

For the analysis of steady-state behavior of BMFLC based methods, an extended version of signal $S_{1}$ is chosen as

$$
S_{4}(t)= \begin{cases}4 \sin (2 \pi 9 t)+2 \sin (2 \pi 11 t) ; & 0 \leq t<60 \\ 2 \sin (2 \pi 7 t)+4 \sin (2 \pi 14 t) ; & 60 \leq t \leq 120\end{cases}
$$

The EEG data set used in the study is from Brain Computer Interface Competition IV [41]. The set contains 


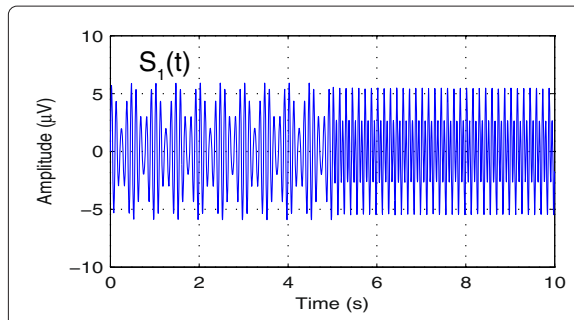

(a1)

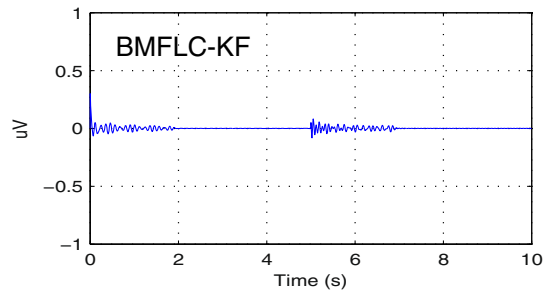

(a2)

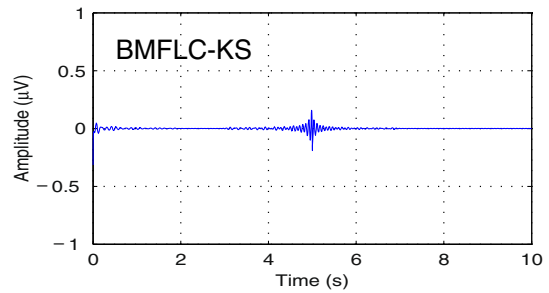

(a3)

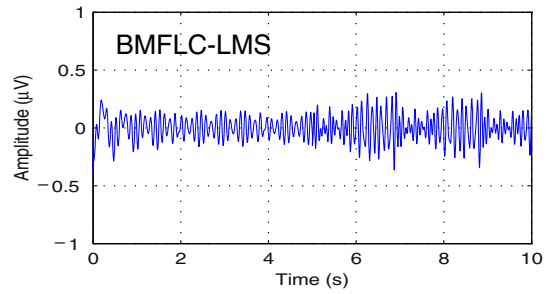

(a4)

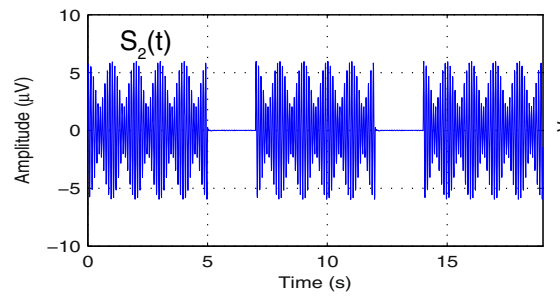

(b1)

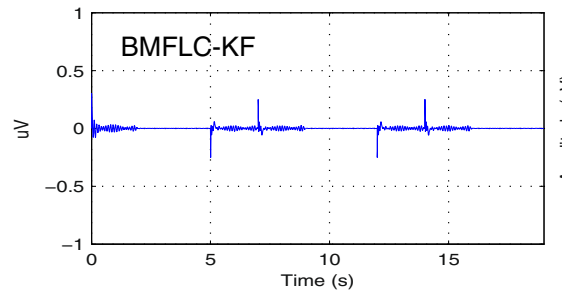

(b2)

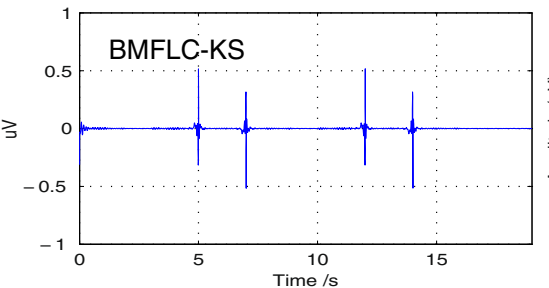

(b3)

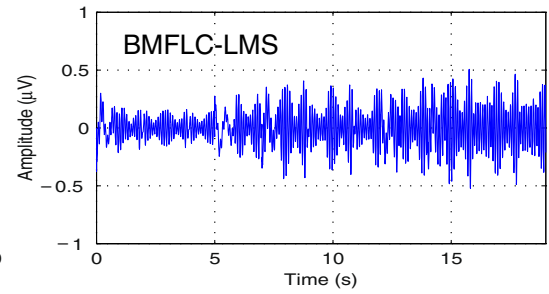

(b4)

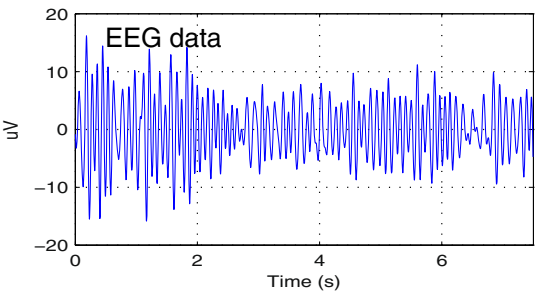

(c1)

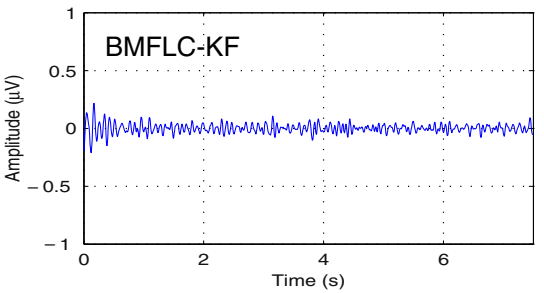

(c2)

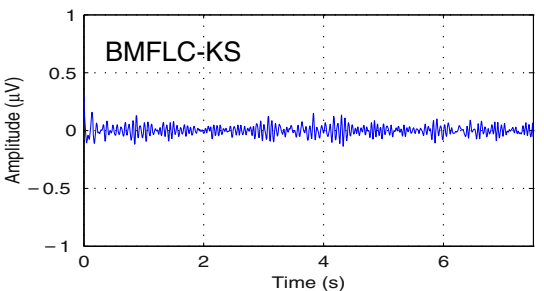

(c3)

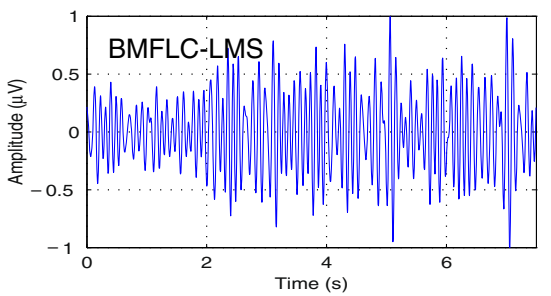

(c4)

Figure 2 Time-domain estimation accuracy of BMFLC, $\boldsymbol{\Delta} \boldsymbol{f}=\mathbf{0 . 5} \mathbf{H z}$. (a) 1), (b1) and (c1) are the synthetic signals $S_{1}(t), S_{2}(t)$ and $E E G$

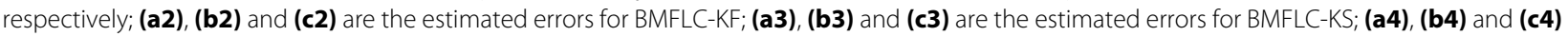
are the estimated errors for BMFLC-LMS.

EEG data of 9 subjects. EEG was recorded from 22 $\mathrm{Ag} / \mathrm{AgCl}$ electrodes sampled at $250 \mathrm{~Hz}$. All signals were recorded monopolarly with the left mastoid as reference and right mastoid as ground. Four classes of cue-based motor imagery tasks were carried out, namely the imagination of movement of the left hand, right hand, both feet and tongue. Each subject data was recorded in 2 sessions on separate days. Each session consists of 6 runs separated by short breaks. One runs consists of 48 trials. During the recording, the subjects sat on a comfortable armchair in front of a computer screen. At the beginning of each trial $(t=0 s)$, a fixation cross appeared on the black screen. Two seconds later, a cue in the form of an arrow pointing either to the left, right, down or up displayed on the screen and lasted 1.25s. The subjects were asked to perform the motor imagery task until the fixation cross disappeared from the screen at $t=6 \mathrm{~s}$. The sequence is shown in Figure 3 (reproduced from [41]). For more details about data collection, see [41]. In this paper the hand movement imagery is considered. For the hand movement imagery, EEG data from the electrodes C3 and C4 placed over the sensorimotor cortex according to $10 / 20$ international system, where the $\mu$-rhythm originates, is selected for analysis. To limit the analysis to $\mu$-band, the data was filtered between $6 \mathrm{~Hz}$ and $14 \mathrm{~Hz}$ by a fifth-order Butterworth bandpass filter.

To quantify the performance of BMFLC based algorithm, we employ the root mean square defined as $R M S(s)=\sqrt{\left(\sum_{k=1}^{k=n}\left(s_{k}\right)^{2} / n\right.}$ where $n$ and $s$ are the number of samples and input signal respectively. $s_{k}$ denotes the 


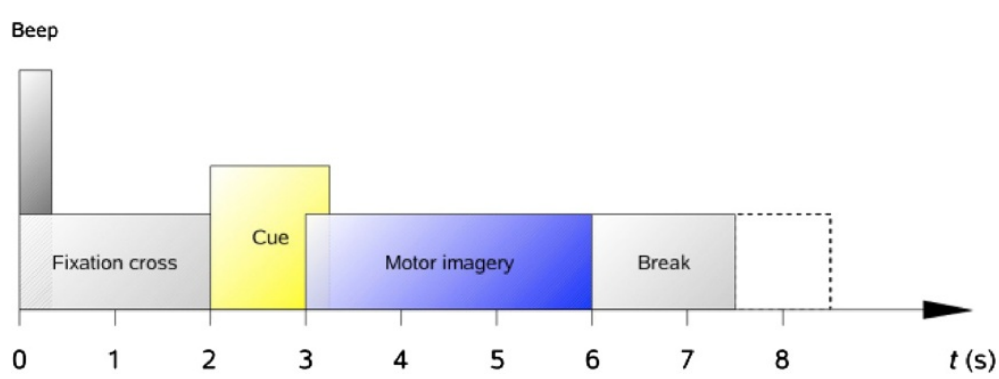

Figure 3 Timing scheme of the experiment.

input signal at time instant $k$. The percentage accuracy is defined as

$$
R M S \%=\frac{R M S(s)-R M S(e)}{R M S(s)} \times 100
$$

where $e$ is the estimation error.

\section{Parameter selection}

For estimation of synthesized signals and EEG, we set the following parameters for BMFLC based algorithms: $f_{1}=6$ $\mathrm{Hz}, \omega_{1}=2 \pi f_{1}, f_{n}=14 \mathrm{~Hz}, \omega_{n}=2 \pi f_{n}$. Frequency spacing is set to be $\Delta f=0.5 \mathrm{~Hz}$ [18]. The weights are initialized with 0 , i.e. $\mathbf{w}_{0}=\mathbf{0}$. For the BMFLC-LMS algorithm, the adaptive gain $\mu=0.035$ is chosen for optimal performance for the corresponding frequency spacing $\triangle f=0.5$ $\mathrm{Hz}$ [18]. The co-relation between the parameter $\mu$ and step-size is discussed in [18].

For implementation of BMFLC-KF/KS, the two parameters, the state noise covariance $\mathbf{Q}$ and measure noise covariance $R$ should be properly tuned. In the following, several experiments are conducted for identification of parameters to achieve better accuracy. To start with, we assume that the state noise covariance is a diagonal matrix in the form of $\mathbf{Q}=q * \mathbf{I}$. Then the parameter $q$ is selected such that the root-mean-square (RMS) error is minimized. In [34], the measurement noise covariance $R$ was estimated online by using the innovation process of the Kalman filter. Then an optimal value for $q$ is selected to minimize the RMS error. Further, the selection of $q$ is also performed for pre-fixed $R$. Experiments are first performed with synthetic signal $S_{1}$ and $S_{2}$ and the corresponding results are shown in Figure 4(a) and 4(b). It shows that when $q>0$, the RMS error is below $3 \%$ when $R$ is estimated online and 1\% for pre-fixed $R$. Based on the result of earlier experiment, we initialize $q=0.05$ and then optimize $R$. The results obtained for signal $S_{1}(t)$ are shown in Figure 4(c). As we vary the value of $R$, the RMS error remains constant. This further shows that the error performance of BMFLC-KF/KS is highly dependent on the selection of $q$.

To further justify the selection of parameters, experiments are conducted with the EEG data. EEG data of all trials of subject 1 are selected. Similar to the earlier, $R$ is estimated in the algorithm, and the $q$ is selected based on the RMS error. Based on the RMS error obtained for all selections of $\mathrm{q}$ and for all trials of the subject \#1, the $95 \%$ confidence interval $(\mathrm{CI})$ is estimated. To obtain reliable estimation of CI, Bootstrap method [42] with 2000 re-sampling is employed and the results are shown in Figure $4(\mathrm{~d})-(\mathrm{e})$. Hence, we select $q=0.01$ and $R=0.01$ for optimal performance.

The STFT and CWT are set to have the same frequency resolution as BMFLC. The window function in STFT is set according to

$$
L=\frac{F_{s}}{\triangle f}
$$

where $L$ denotes the length of the window function, $F_{S}$ is the sampling frequency and $\triangle f=\Delta \omega / 2 \pi$. The step size of STFT is $1 / F_{s}$. For the wavelet-based TFR method, the Morlet wavelet is employed with $\omega_{0}=6$ to offer good trade-off between temporal and spectral resolutions [22]. A total of 17 scales are calculated in this paper, which are equally spaced within the range of $6 \mathrm{~Hz}$ to $14 \mathrm{~Hz}$ with the same frequency gap employed in BMFLC and STFT. The wavelet scale is transformed to Fourier frequency with (3).

\section{Results}

In this section, we provide comprehensive analysis of all the five methods, BMFLC-LMS, BMFLC-KF, BMFLC-KS, STFT and CWT for synthetic and EEG data sets discussed in earlier section.

\section{Estimation accuracy}

To compare the estimation accuracy of BMFLC based methods, the estimated signal together with the estimation error for synthesized signals $(23,24)$ and single trial EEG data (C3 electrode for right hand movement imagery) are shown in Figure 2.

In Figure 2(a2) and Figure 2(b2), error can be observed at the transition points for BMFLC-KF. By comparing the results of BMFLC-LMS in Figure 2(a4) and BMFLC-KF/KS in Figure 2(a2) and (a3), it is clear that 


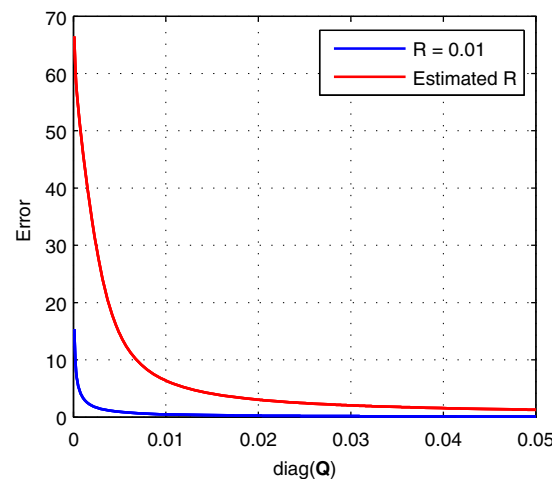

(a)

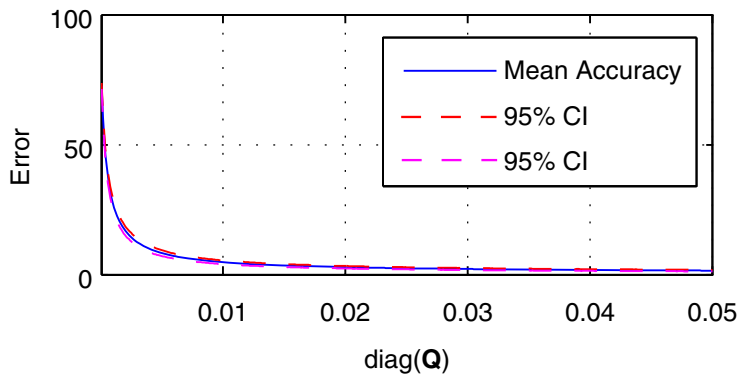

(d)

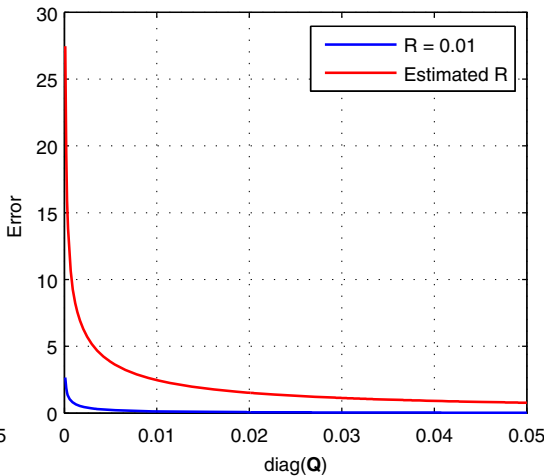

(b)

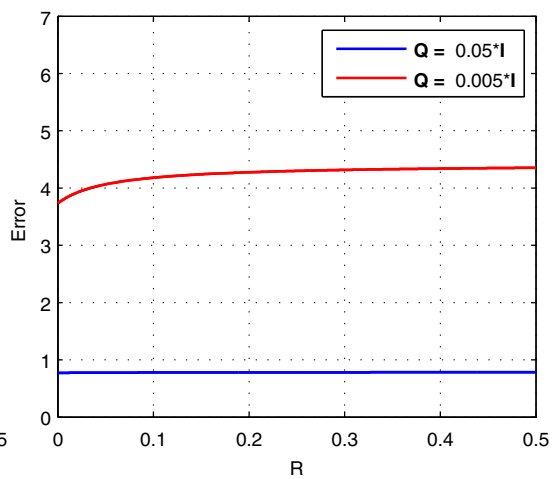

(c)

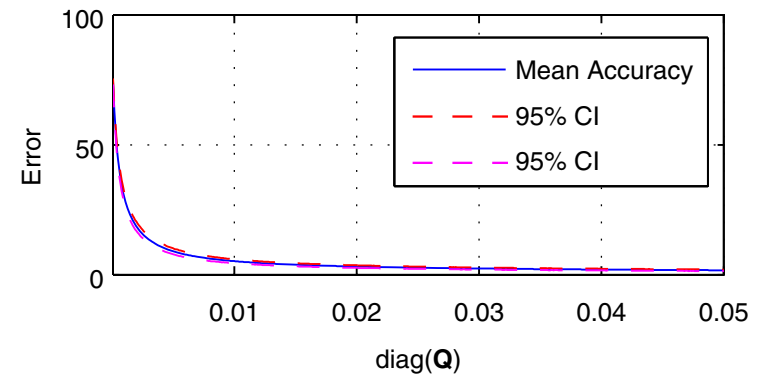

(e)

Figure 4 Parameter tuning for Kalman filter. (a) Parameter $q$ selection based on $S_{1}(t)$; (b) Parameter $q$ selection based on $S_{2}(t)$; (c) Parameter $R$ selection with $S_{1}(t)$ for fixed q; (d) Subject \#1 C3(all trials); (e) Subject \#1 C4(all trials).

the estimation accuracy depends on the adaptive algorithm. For the EEG data, BMFLC-KF/KS performed better compared to BMFLC-LMS as shown in Figure 2(c4).

For BMFLC-KS, the error is backward averaged to obtain the smoothed estimation. Since the system transition matrix is modelled as identity matrix, the accuracy of the algorithm cannot be improved with Kalman-smoother [36]. However, we can observe from Figure 2(b2) and Figure 2(b3) that the transient performance can be improved.

The RMS\% error for all methods is tabulated in Table 1. The results indicate that the proposed method accurately models both the synthesized signals and EEG data. For a large EEG data set, the accuracy remained nearly constant with small variation.

Table 1 Estimation accuracy of BMFLC based methods

\begin{tabular}{|c|c|c|c|c|c|c|}
\hline \multirow{2}{*}{ Methods } & \multicolumn{6}{|c|}{ Signal } \\
\hline & $S_{1}(t)$ & $S_{2}(t)$ & $S_{3}(t)$ & $\mathrm{EEG}^{a}$ & C3_RH_All & C4_RH_Allb \\
\hline BMFLC-KF & 99.47 & 99.39 & 99.49 & 99.22 & $99.19 \pm 0.14$ & $99.03 \pm 0.20$ \\
\hline BMFLC-KS & 99.53 & 99.12 & 99.44 & 99.19 & $98.87 \pm 0.27$ & $98.80 \pm 0.34$ \\
\hline BMFLC-LMS & 96.60 & 94.26 & 96.68 & 93.68 & $93.42 \pm 0.69$ & $93.55 \pm 0.71$ \\
\hline
\end{tabular}

To analyze the effect of frequency gap on estimation accuracy, the RMS\% accuracy for all methods for various choices of frequency gap is computed with 68 trials of EEG data (C3_RH_All). Table 2 shows that the choice of frequency gap does not effect the accuracy of the estimation. However, the selection of frequency gap affects the frequency tracking that can be obtained from BMFLC. This issue will be discussed in the following section.

\section{Temporal and spectral resolution: comparison of all five methods}

The time-frequency representation of two synthesized signals together with the single trial EEG data obtained with STFT, CWT and BMFLC based methods are shown in Figure 5. The leakage effects of STFT and CWT can be clearly identified (Figure 5(a5), (b5), (a6) and (b6)) in the adjacent frequency components. The colorbar represents the absolute amplitude of corresponding

Table 2 Estimation accuracy for different frequency gaps

\begin{tabular}{lcccc}
\hline \multirow{2}{*}{ Methods } & \multicolumn{4}{c}{ Frequency gap in BMFLC } \\
\cline { 2 - 5 } & $\Delta f=0.1 \mathrm{~Hz}$ & $\Delta f=0.2 \mathrm{~Hz}$ & $\Delta f=0.5 \mathrm{~Hz}$ & $\Delta f=1 \mathrm{~Hz}$ \\
\hline BMFLC-KF & $99.80 \pm 0.03$ & $99.63 \pm 0.05$ & $99.19 \pm 0.14$ & $98.50 \pm 0.11$ \\
\hline BMFLC-KS & $99.20 \pm 0.10$ & $99.03 \pm 0.15$ & $98.87 \pm 0.27$ & $99.07 \pm 0.15$ \\
\hline BMFLC-LMS & $96.93 \pm 0.22$ & $95.80 \pm 0.40$ & $93.42 \pm 0.69$ & $96.16 \pm 0.77$ \\
\hline
\end{tabular}




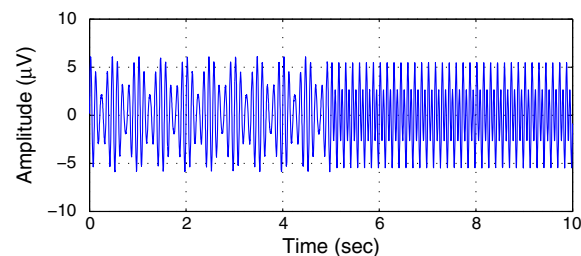

(a1) $\mathrm{S}_{1}(\mathrm{t})$

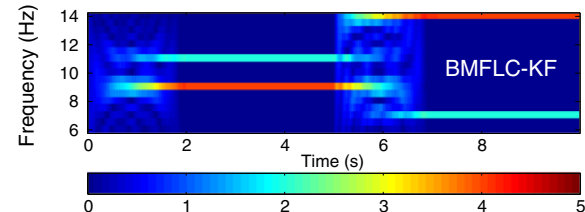

(a2)

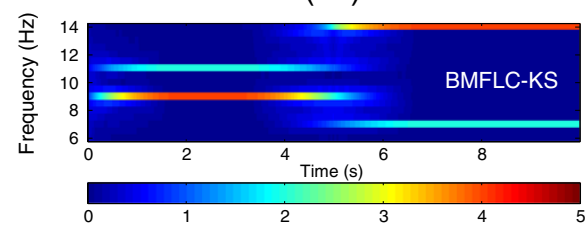

(a3)

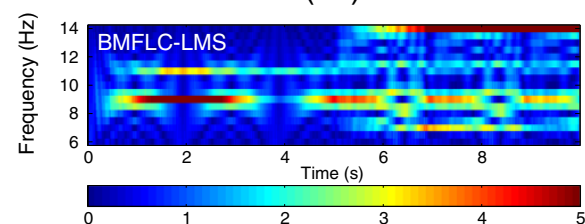

(a4)

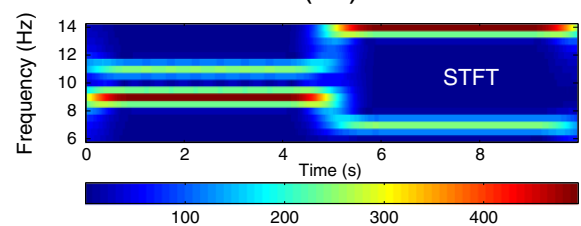

(a5)

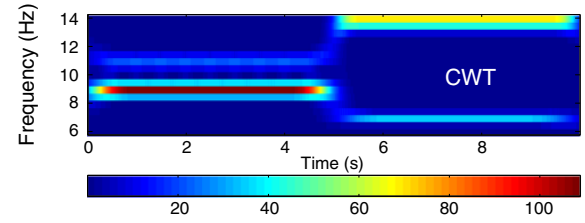

(a6)

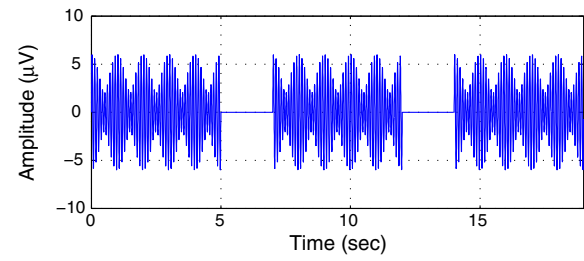

(b1) $\mathrm{S}_{2}(\mathrm{t})$

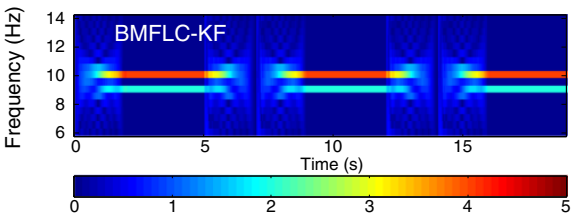

(b2)

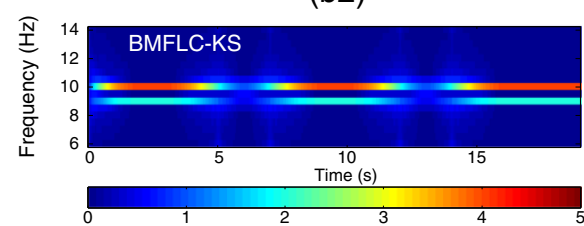

(b3)

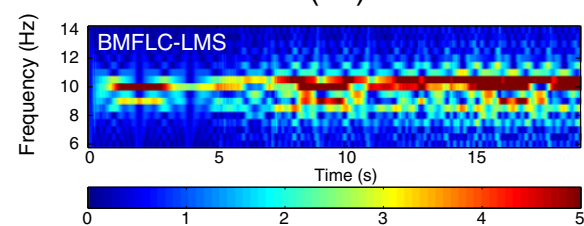

(b4)

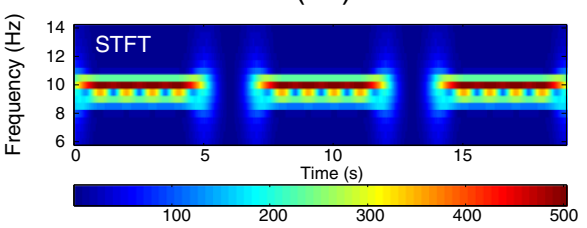

(b5)

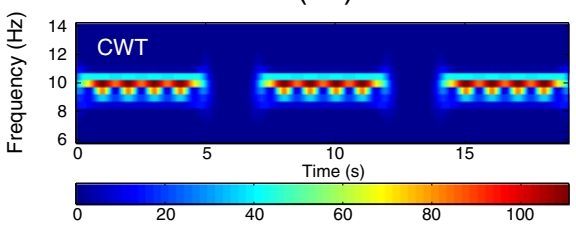

(b6)

Figure 5 Time-Frequency mapping for synthesized signals, $\boldsymbol{S}_{\mathbf{1}}(\boldsymbol{t})$ and $\boldsymbol{S}_{\mathbf{2}}(\boldsymbol{t}), \boldsymbol{\Delta} \boldsymbol{f}=\mathbf{0 . 5} \mathrm{Hz}$. (a) 1$)$ and $(\mathbf{b} \mathbf{1})$ are the synthetic signal $S_{1}(t)$ and $S_{2}(t)$ respectively; (a2) and $\mathbf{( b 2 )}$ are the time-frequency mappings obtained with BMFLC-KF; (a3) and (b3) are the time-frequency mappings obtained with BMFLC-KS; (a4) and (b4) are the time-frequency mappings obtained with BMFLC-LMS; (a5) and (b5) are the time-frequency mappings obtained with STFT; (a6) and (b6) are the time-frequency mappings obtained with CWT.

frequency components in the figure for BMFLC-LMS, BMFLC-KF/KS. By construction, BMFLC models the signal into individual frequency components and hence the leakage effect that occurs in STFT and CWT can be mitigated.

BMFLC-KF provides accurate spectral estimation as shown in Figure 5(a2) and (b2). However, when there is a sudden change in the frequency, BMFLC-KF requires an adapting period for tracking the spectral changes in the signal. Although the estimation accuracy is high, there is some disturbance at the frequency transition at $5 \mathrm{sec}$ as seen in Figure 5(a2) and (b2). As the amplitude weights of BMFLC-KF are initialized with zero, BMFLC-KF requires an initial adaptation period. This is mainly due to the random walk model employed for the state transition in BMFLC. By comparing Figure 5(a2) with Figure 5(a3) and 
Figure 5(b2) with Figure 5(b3), the estimation of smoother relays on the information provided by the Kalman filter, and operates backward to smooth the errors in the estimation. It is clear that the BMFLC-KS provides improved spectral estimation.

Another factor that affects the spectral estimation is the selection of frequency gap $\Delta f$. To study the sensitivity of the method, synthesized signal $S_{3}(t)$ is employed and the results for various $\Delta f$ are shown in Figure 6. When source signal has several frequency components located closely in the spectral domain, STFT provides better spectral estimation compared to CWT. It is also clear that, with STFT the estimated amplitude is distorted. A frequency gap of $0.2 \mathrm{~Hz}$ is employed for the analysis with BMFLC based methods. For a frequency gap $\Delta f=0.1 \mathrm{~Hz}$ and $0.2 \mathrm{~Hz}$, spectral estimation obtained with BMFLC-KF/KS is better compared to STFT. With BMFLC-KS the initial adaptation period is reduced and the improved performance can be seen in Figure 6(d). As the source signal $S_{3}(t)$ has closely spaced frequencies, the results for BMFLC with frequency gap $1 \mathrm{~Hz}$ are not accurate as shown in Figure 6(h). Furthermore, the number of frequency weights in BMFLC based methods can affect the amplitude estimation. As shown in Figure 6(g), when $\Delta f=0.1$ $\mathrm{Hz}$ gap is used, the estimated amplitude is smaller compared to the actual amplitude of the synthesized signal $S_{3}(t)$. These results clearly shows that the an appropriate

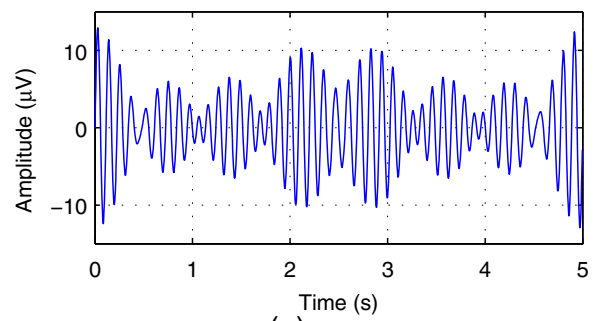

(a) $S_{3}(t)$

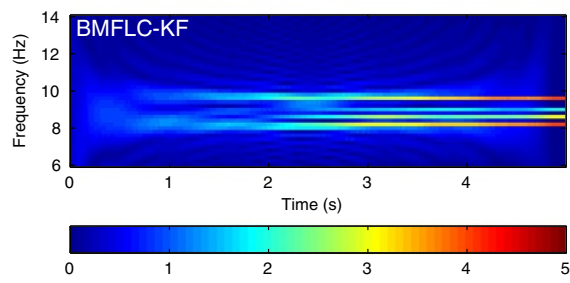

(c) $\Delta f=0.2 \mathrm{~Hz}$

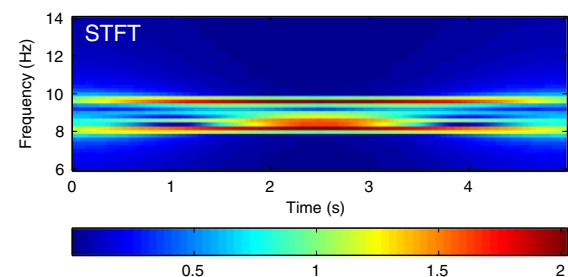

(e) STFT

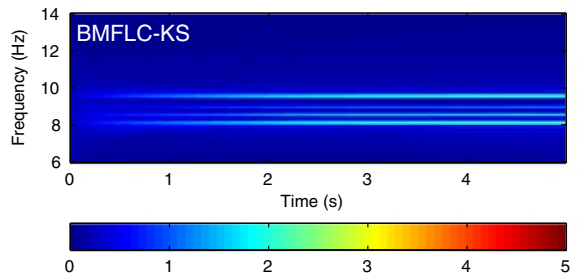

(g) $\Delta f=0.1 \mathrm{~Hz}$

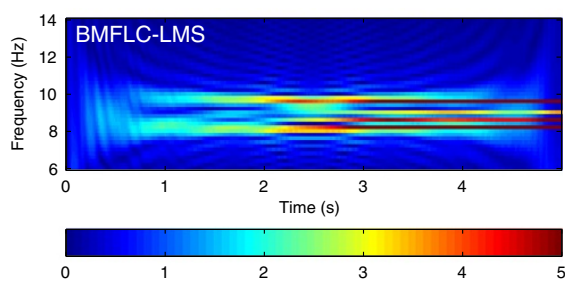

(b) $\Delta f=0.2 \mathrm{~Hz}$

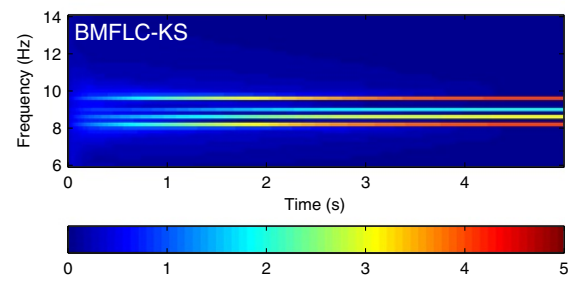

(d) $\Delta t=0.2 \mathrm{~Hz}$

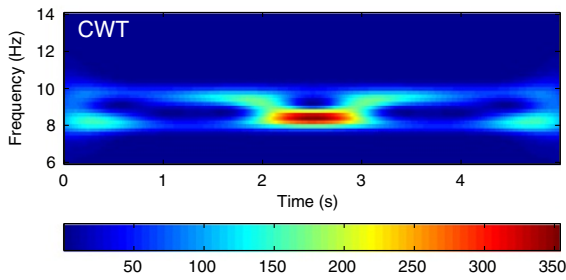

(f) CWT

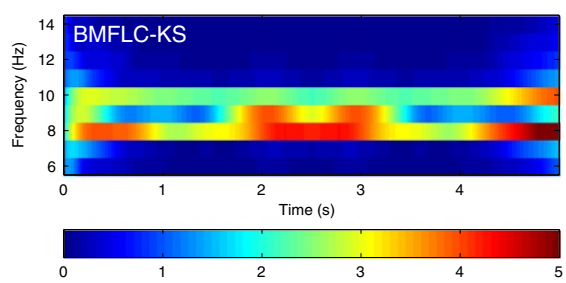

(h) $\Delta f=1 \mathrm{~Hz}$

Figure 6 Time-frequency mappings of various methods for synthetic signal $S_{\mathbf{3}}(\boldsymbol{t})$. (a) Synthetic signal $S_{3}(t)$; (b) Time-frequency mapping of BMFLC-LMS with frequency spacing $\Delta f=0.2 \mathrm{~Hz}$; (c) Time-frequency mapping of BMFLC-KF with frequency spacing $\Delta f=0.2 \mathrm{~Hz}$; (d) Time-frequency mapping of BMFLC-KS with frequency spacing $\Delta f=0.2 \mathrm{~Hz}$; (e) Time-frequency mapping of STFT; (f) Time-frequency mapping of CWT; (g) Time-frequency mapping of BMFLC-KS with frequency spacing $\Delta f=0.1 \mathrm{~Hz}$; (h) Time-frequency mapping of BMFLC-KS with frequency spacing $\Delta f=1 \mathrm{~Hz}$ 
frequency gap should be selected for accurate spectral estimation.

The inadequacy of CWT for the synthetic signal $S_{3}(t)$ in Figure 6(f) is mainly due to the parameter selection of the mother wavelets. The bigger the $\omega_{0}$ in Morlet wavelets, the better frequency resolution can be obtained. By proper tuning of $\omega_{0}$, an improved spectral resolution can be obtained for $S_{3}(t)$ as shown in Figure 7(a). However, for the same parameter selection, the CWT for the signal $S_{2}(t)$ is shown in Figure 7(b) and the temporal resolution is compromised. It is clear that if high accuracy is required in the spectral domain, accuracy in the temporal resolution cannot be guaranteed at the same time. Comparing the above results, BMFLC method provides better frequency resolution without loss of temporal resolution.

Although the amplitude accuracy of BMFLC-LMS is high, its corresponding frequency components cannot adjust to the sudden changes in the frequency characteristics of the signal. The weights of BMFLC-LMS requires longer duration to track the changes in the frequency characteristics of the signal. To highlight the problem, synthesized signal $S_{4}(t)$ is employed. Time-frequency maps for BMFLC-LMS, BMFLC-KF/KS are shown in Figure 8(a). Individual weights of BMFLC are shown in Figure 8(b)-(d). It can be clearly seen in Figure 8(d) that the frequency weights of BMFLC-LMS require more time to settle to steady-state. Whereas BMFLC-KF/KS weights settle to correct frequency values immediately as shown in Figure 8(c)-(d). When the frequency components in signal $S_{4}$ change at $60 \mathrm{~s}$, the previous settled frequency weights slowly decreases to 0 as the new frequency components gradually increase. BMFLC-KF/KS can track the sudden changes in frequency, whereas the corresponding weights in BMFLC-LMS does not settle. This clearly highlight the inadequacy of the BMFLC-LMS for extracting fast changing frequency characteristics in the signal.

\section{Computational complexity}

In order to study the real-time applicability, the computational complexity of the TFR methods is presented. The difference between BMFLC-KF and non-parametric methods (CWT and STFT) is that they require sufficient length of input samples to be stored in order to provide the time-frequency mapping. Therefore the computational complexity of these methods relies on the length of the input data.

On the other hand, the BMFLC-KF updates the estimation at every sample. Therefore the computational complexity only relies on the observation and state-space dimensions of the Kalman filter and is independent of the input data length. The computational complexity of Kalman filter is given by $O\left(3 l^{2}\right)$, where $l$ and $n$ are observation and state-space dimensions respectively [43]. For STFT, the input data is first multiplied with a window function followed by FFT [44]. Hence the computational complexity of STFT is $O\left(\mathrm{Nlog}_{2} N\right)$, where $N$ is the length of the data being analyzed. The inverse Fourier transform for CWT requires three FFT's for a signal with length $N$. Hence the computational complexity of CWT can be computed as $\mathrm{O}\left(3 \mathrm{~N} \log _{2} N\right)$. For comparison, the operations required for all methods are tabulated in Table 3.

In $\mathrm{BCIs}$, the feature required for generating a control command is extracted every $500 \mathrm{~ms}[16,45]$. Let us consider the sampling frequency as $F_{s}=512 \mathrm{~Hz}$ and frequency resolution as $\Delta f=0.5 \mathrm{~Hz}$ [18]. For the given $\Delta f$, the order of BMFLC-KF can be obtained as $n=\frac{f_{n}-f_{1}}{\Delta f} \times 2$. In order to maintain the same frequency resolution in STFT and FFT with BMFLC, the data is padded with zeros in order to have sufficient data length (28) for analysis. For quantitative comparison, the operations required for all the methods are for a given data length is also provided in Table 3. The computational complexity of FFT [45] is also discussed in the Table. This comparison quantifies the computational complexity of the algorithms for real-time implementation. BMFLC-KF has comparatively lower computational requirement compared to other methods.

The computational complexity of BMFLC-KF depends on the dimensions of the state-space which grows linearly as the frequency gap $\Delta f$ decreases. The computational requirement of BMFLC-KF for various $\Delta f$ are given in Table 4. For a small frequency gap $\Delta f=0.1$ or 0.2 , the computational complexity increases and the method becomes impractical for real-time applications. However,

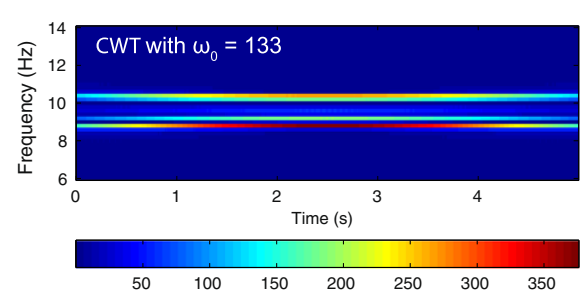

(a) CWT for $\mathrm{S}_{3}(\mathrm{t})$

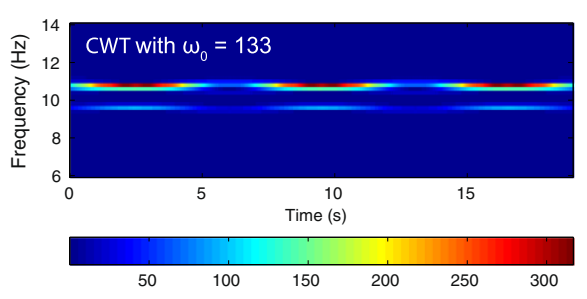

(b) CWT for $\mathrm{S}_{2}(\mathrm{t})$

Figure 7 Modified CWT for synthetic signals $\boldsymbol{S}_{\mathbf{2}}(\boldsymbol{t})$ and $\boldsymbol{S}_{\mathbf{3}}(\boldsymbol{t})$. (a) Time-frequency mapping of CWT for $S_{3}(t)$ with $\omega_{0}=133$; (b) Time-frequency mapping of $C W T$ for $S_{2}(t)$ with $\omega_{0}=133$. 

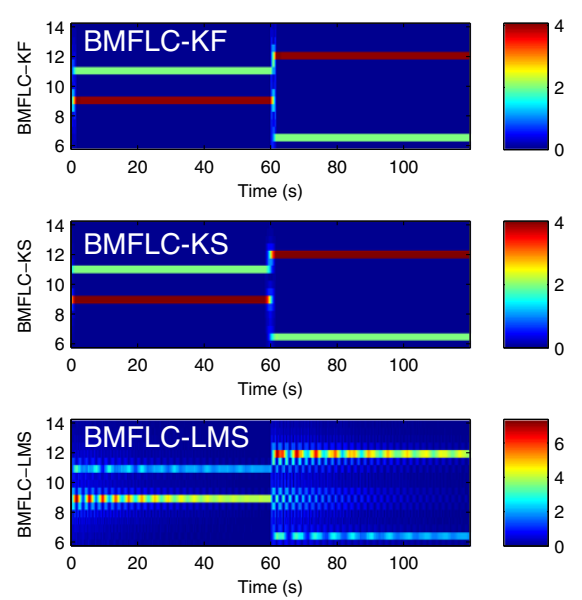

(a) Time-Frequency Mappings

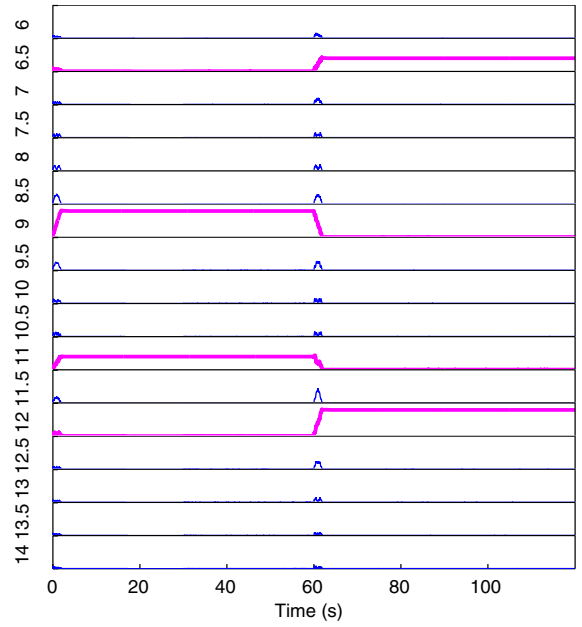

(c) Weights Profile of BMFLC-KF

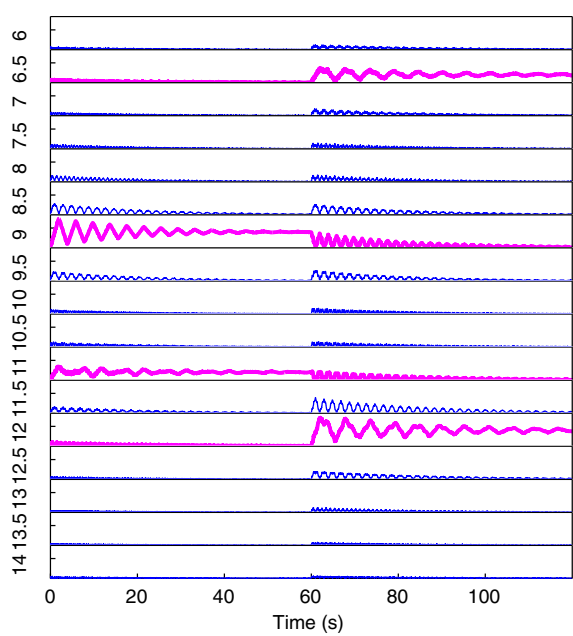

(b) Weights Profile of BMFLC-LMS

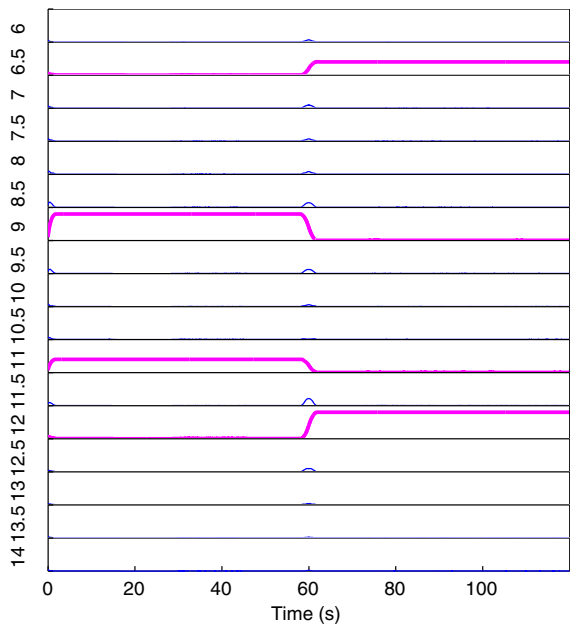

(d) Weights Profile of BMFLC-KS

Figure 8 Estimation performance for synthetic signal $\boldsymbol{S}_{\mathbf{4}}(\boldsymbol{t})$. (a) Time-frequency mappings for BMFLC-KF, BMFLC-KS and BMFLC-LMS; (b) Estimated weights of BMFLC-LMS; (c) Estimated weights of BMFLC-KF; (d) Estimated weights of BMFLC-KS.

for EEG applications with an appropriate frequency gap, i.e. $\triangle f=0.5 \mathrm{~Hz}$ as in [18], the BMFLC-KF can be used for BCI applications to provide better spectral resolution with less computation as compared to STFT and CWT.

\section{ERD detection}

In order to test the efficiency of the proposed algorithm for BCI applications, we apply all five TFR methods to

Table 3 Computational complexity

\begin{tabular}{lcccc}
\hline & FFT $^{a}$ & STFT $^{a}$ & CWT $^{a}$ & BMFLC-KF $^{\boldsymbol{b}}$ \\
\hline Notation & $O\left(N_{1} \log _{2} N_{1}\right)$ & $O\left(N \log _{2} N_{1}\right)$ & $O\left(3 N_{2} \log _{2} N_{2}\right)$ & $O\left(3 / n^{2}\right)$ \\
\hline Operations $^{c}$ & 10240 & 10240 & 6144 & 3072 \\
\hline
\end{tabular}

${ }^{a} N$ is the data length; $N_{1}=1024 ; N_{2}=256$;

${ }^{b} /$ and $n$ are the observation and filter orders respectively.

${ }^{c} F_{s}=512 \mathrm{~Hz}, \Delta f=0.5 \mathrm{~Hz}$. the EEG data for ERD detection. ERD detection can be identified in two ways. It can be either seen as an energy decreasing with respect to the experiment cue in the timefrequency mapping or as an energy percentage change with respect to a reference period (a pre-defined period before the experiment cue onset). The percentage value denoted as $E R D_{j}^{f}$ is defined similar to [42] as

$$
A_{j}^{f}=\frac{1}{N-1} \sum_{j=1}^{N}\left(\mathbf{w}_{i, j}^{f}-\overline{\mathbf{w}}_{j}^{f}\right)^{2}
$$

Table 4 Computational complexity of BMFLC-KF

\begin{tabular}{|c|c|c|c|c|}
\hline BMFLC-KF & $\Delta f=0.2 \mathrm{~Hz}$ & $\Delta f=0.4 \mathrm{~Hz}$ & $\Delta f=0.5 \mathrm{~Hz}$ & $\Delta f=1 \mathrm{~Hz}$ \\
\hline Operations & 19200 & 4800 & 3072 & 768 \\
\hline
\end{tabular}




$$
\begin{aligned}
& R^{f}=\frac{1}{k} \sum_{r_{0}}^{r_{0}+k} A_{j}^{f} \\
& E R D_{j}^{f}=\frac{A_{j}-R}{R} \times 100 \%
\end{aligned}
$$

where $\mathbf{w}_{i, j}^{f}$ are the estimated weights from time-frequency decomposition algorithm at $j$ th sample of the $i$ th trial of frequency $f, \overline{\mathbf{w}}_{j}^{f}$ is the mean of weights over all trials and $N$ is the number of trials/subject. $R^{f}$ is average power in the pre-defined reference period $\left[r_{0}, r_{0}+k\right]$. The reference period is generally the period before the cue is onset [9]. In line with earlier works $[9,32]$, we select the reference period as 0 to $1.5 \mathrm{~s}$ before the cue onset (cue at 2 sec) for calculation of ERD. The time-frequency map for ERD is obtained by combining all the row vectors $E R D^{f}=$ $\left[E R D_{1}^{f} E R D_{2}^{f} \cdots E R D_{m}^{f}\right]$, with $m$ being the number of data samples in a given trial. The obtained ERD \% is the average over all trials of a subject.

The ERD detection of the corresponding electrode location and all trials for 3 subjects (Subject 1 right hand imagery, Subject 3 right hand imagery and Subject 7 right hand imagery) is shown in Figure 9. ERD pattern can be identified for all the three subjects in different frequency bands for all the methods. Comparing the results of BMFLC-KF (Figure 9(a1)-(c1)) with STFT (Figure 9(a3)(c3)) and CWT (Figure 9(a4)-(c4)), there is a small delay in the event transition in the ERD pattern. Whereas BMFLC-KS (Figure 9(a2)-(c2)) shows similar performance compared to STFT and CWT. It is clear that BMFLC-KF requires additional $0.5 \mathrm{~s}$ for the settlement of frequency weights (as depicted in the Figure 9(a1)-(c1) during the initial $0.5 \mathrm{~s})$.

To statistically validate the performance, bootstrap with 2000 times re-sampling is used to estimate the 95\% confidence interval for obtained ERD\%. As stated in [42], if both confidence values of an ERD show same sign then it can be considered as significant. The bootstrap test shows that the obtained ERD mapping is significant for all methods.

In order to quantify the ERD, the difference between maximal ERD percentage value and minimal ERD percentage value averaged over all frequency components is analyzed. The results are shown in Figure 10. A z-test has been applied to check whether the results are significantly

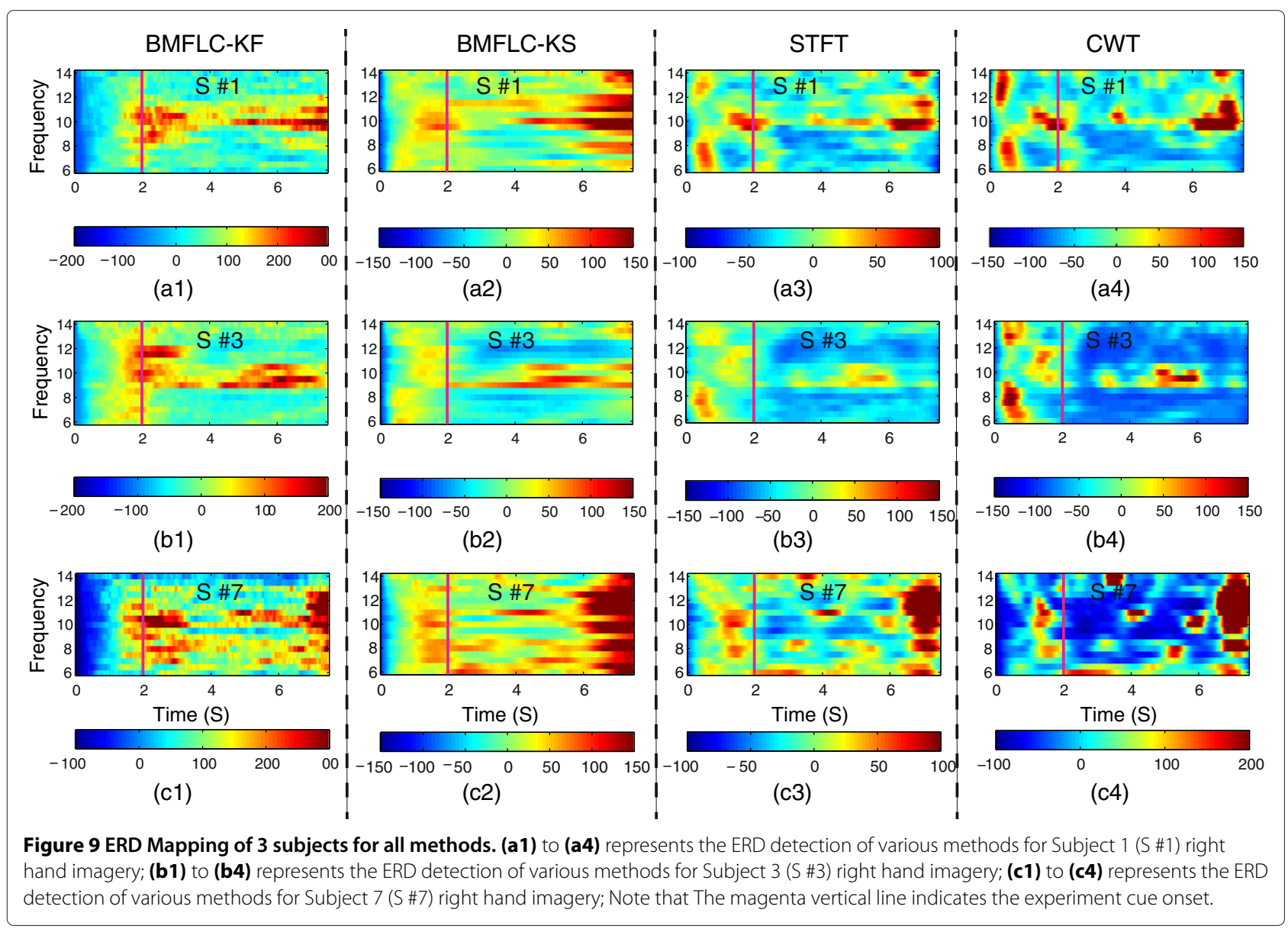




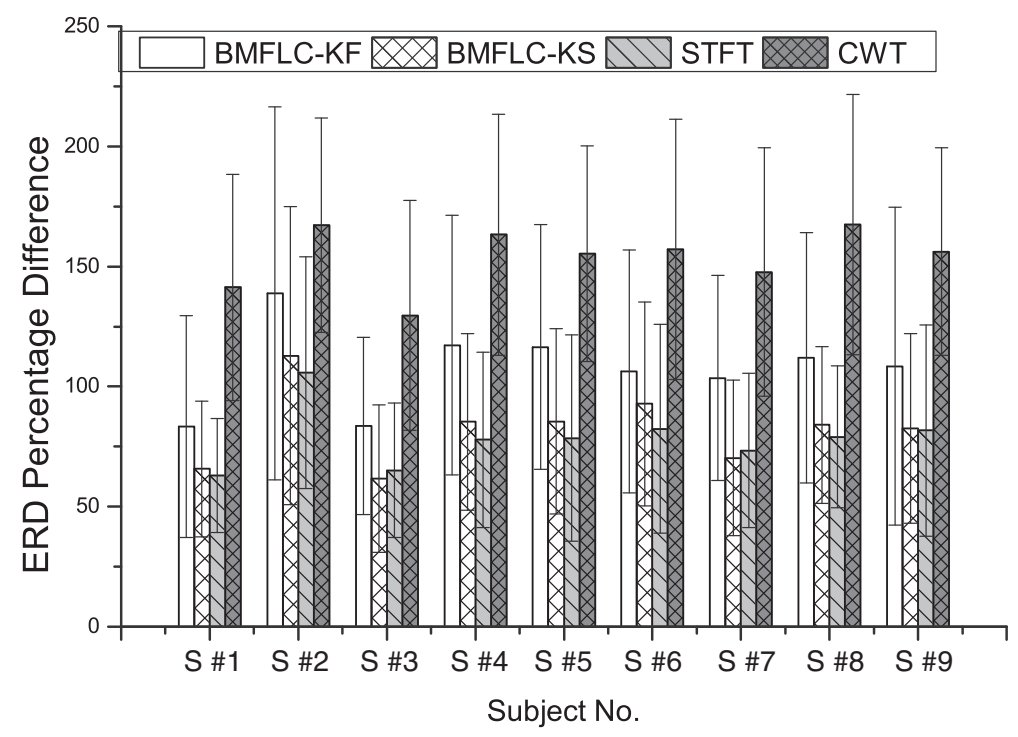

Figure 10 ERD detection comparison for all subjects of right hand imagery.

different among methods. For all the subjects, the CWT shows the highest difference in ERD detection in mean value. The BMFLC-KF performs better than STFT over all the subjects $(z=11.12, p<0.01)$ and it also outperforms BMFLC-KS $(z=15.01, p<0.01)$. The CWT provides the best performance compared to all the four methods.
To highlight the applicability of the proposed method for BCI applications, single trial ERD pattern for subject 3 right hand imagery from C3 location (shown in Figure 11(a1)) was analyzed. ERD for a single trial data can be visually identified as an energy decreasing phenomenon after cue is onset in Figure 11 for all the

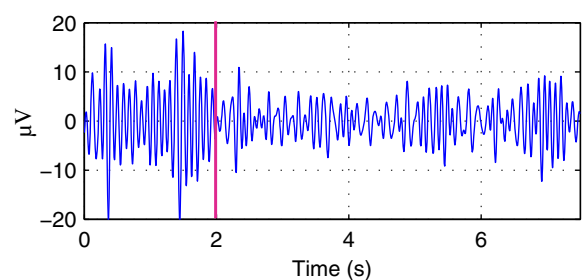

(a1) Single Trial EEG data of S\#3

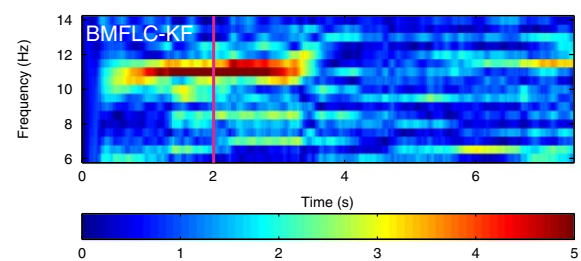

(b2)

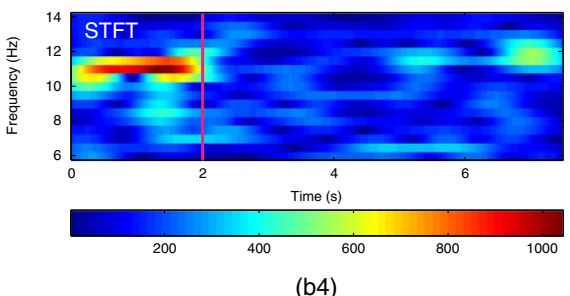

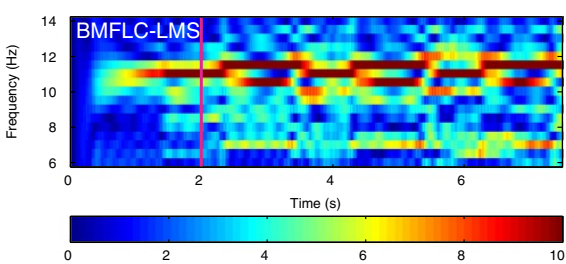

(b1)

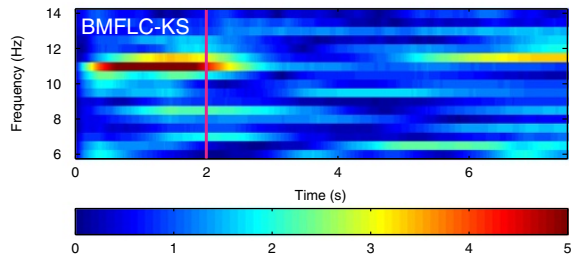

(b3)

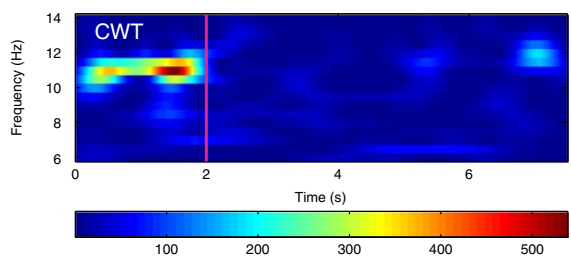

(b5)

Figure 11 ERD detection for Subject 3 in one trial. The magenta vertical line indicates the experiment cue onset. (a1) Raw EEG signal; (b1) Time-frequency mapping with BMFLC-LMS; (b2) Time-frequency mapping with BMFLC-KF; (b3) Time-frequency mapping with BMFLC-KS; (b4) Time-frequency mapping with Short-time Fourier Transform; (b5) Time-frequency mapping with Continuous-wavelet Transform. 
methods. Since the motor sensory ERD only exists before the movement onset, this phenomenon is extremely sensitive to time delay.

By comparing the results in Figure 11(b2)-(b5), ERD can be observed in the frequency range of $10 \mathrm{~Hz}-12 \mathrm{~Hz}$ in all the methods except BMFLC-LMS. Within these methods, STFT (Figure 11(b4)) and CWT (Figure 11(b5)) offer the best temporal accuracy. On the other hand, BMFLC$\mathrm{KF} / \mathrm{KS}$ also provides narrower band similar to CWT. By comparing the results of Figure 11(b1) and (b2), the adaptation period in Kalman filter can be decreased with the smoother procedure. Also, the frequency transitions in the spectral domain can be accurately estimated in the time-frequency mapping for $\mathrm{BCI}$ applications with the proposed method.

\section{Discussion}

Although the proposed method ensures high accuracy for all choices of $(\Delta f)$, an optimal selection of $(\Delta f)$ is required for real-time implementation. For EEG, a frequency gap of $0.5 \mathrm{~Hz}$ is optimal [18] and ensures accurate spectral estimation. However, for off-line analysis a small $(\Delta f)$ can be selected. The comparative study conducted on computational complexity of the TFRs shows that the proposed method has lower computational demand for a bandlimited signal. Hence, the proposed method is more suitable for accurate spectral estimation in a narrow frequency band.

While the proposed method is only discussed for a narrow frequency band in this paper, the method can still be applied for a wide frequency band. Multiple BMFLCs can be implemented in parallel by dividing the wide band into small narrow bands to ensure stability and accuracy for the algorithm. However, if the band of interests is too wide or the frequency resolution for each sub-band is too high, traditional methods would be more appropriate. A wide frequency band increases the computational requirement in BMFLC. As we confine our study to a narrow frequency band, the CWT could not provide better performance compared to STFT as the frequency resolution in CWT is scaled in the narrow band.

Heisenberg uncertainty exists in all the methods. Even though the estimation accuracy is high, the proposed method requires an additional time for the frequency weights to settle, as both amplitude and frequency cannot be estimated at the same time. The uncertainty on frequency can be clearly seen at the sudden frequency or amplitude transition. For EEG signals, an additional $0.5 \mathrm{~s}$ is required for the frequency weights to settle. However, it only occurs at the start of the estimation process. This initial estimation delay is inherent for all adaptive based methods and can be improved with proper initialization. The lower computational complexity of the proposed method can offset for the delay caused in the estimation compared to traditional methods. Comparatively, the CWT has the best performance in ERD detection. However, the implementation of CWT method can be a problem for real-time applications.

Although only the ERD detection is considered in this paper as application, the proposed algorithm is applicable for any bandlimited signal estimation. As ERD and ERS (event related synchronization) lies in a specific frequency bands, the proposed method can be applied for ERD and ERS detection simultaneously by employing multiple BMFLC's in parallel. Since the weights in the BMFLC are directly related to the real amplitudes of the individual frequency components, the algorithm can utilized where an accurate amplitude estimation of a specific frequency component is required.

\section{Conclusions}

In this research, the performance of existing BMFLC-LMS is improved by incorporating a Kalman filter. A comparison study of the BMFLC based methods with STFT and CWT is performed with both synthetic and real EEG data. The results indicate that the BMFLC-KF/KS can be used as an alternative time-frequency analysis methods for band-limited signals. As most of the frequency-based BCI applications rely on amplitude features in a fixed frequency band ( $\mu$ rhythm) for classification, BMFLCKF can be directly applied to most existing BCI systems. With the linear model employed, optimal estimation can be obtained with the Kalman filter. Thus the proposed method can provide an accurate time-frequency mapping with less computational complexity as compared to STFT and CWT for real-time applications. The results also show that the BMFLC-KS can provide more accurate time-frequency representation for off-line analysis.

\section{Competing interests}

The authors declare that they have no competing interests.

\section{Authors' contributions}

The work was carried out as part of the Ph.D study by Mr. Yubo Wang under the guidance of Dr. Kalyana Veluvolu and Dr. Minho Lee. All authors read and approved the final manuscript.

\section{Acknowledgements}

This research was supported by the Basic Science Research Program through the National Research Foundation of Korea (NRF) funded by the Ministry of Education, Science and Technology (Grant No. 2011-0023999).

Received: 22 May 2013 Accepted: 19 November 2013

Published: 25 November 2013

\section{References}

1. Buzsaki G, Draguhn A: Neuronal oscillations in cortical networks. Science 2004, 304:1926-1929.

2. Olufsen MS, Whittington MA, Camperi M, Kopell N: New roles for the gamma rhythm: population tuning and preprocessing for the beta rhythm. J Comput Neurosci 2003, 14:33-54.

3. Dimitriadis SI, Laskarisb NA, Tsirkac V, Vourkasd M, Micheloyannisc S: What does delta band tell us about cognitive processes: A mental calculation study. Neurosci Lett 2010, 483:11-15. 
4. Pfurtscheller G, Muller-Putz GR, Scherer R, Neuper C: Rehabilitation with brain-computer interface systems. Computer 2008, 41:1-8.

5. Wolpaw JR, Birbaumer N, McFarland DJ, Pfurtscheller G, Vaughana TM: Brain computer interfaces for communication and control. Clin Neurophysiol 2002, 113:767-791

6. Blankertz B, Losch F, Krauledat M, Dornhege G, Curio G, Muller KR: The Berlin brain-computer interface: accurate performance from first-session in BCl-Naive subjects. IEEE Trans Biomed Eng 2008, 55:2452-2462.

7. Ferreira A, Celeste WC, Cheein FA, Bastos-Filho TF, Sarcinelli-Filho M, Carelli R: Human-machine interfaces based on EMG and EEG applied to robotic systems. J Neuroeng Rehabil 2008, 5:10.

8. Liu G, Zhang D, Meng J, Huang G, Zhu X: Unsupervised adaptation of electroencephalogram signal processing based on fuzzy C-means algorithm. Int J Adaptive Control Signal Process 2012, 26:482-495.

9. Pfurtscheller G, Silva FLD: Event-related EEG/MEG synchronization and desynchronization: basic principles. Clin Neurophysiol 1999, 110:1842-1857.

10. Prasad G, Herman P, Coyle D, McDonough S, Crosbie J: Applying a brain-computer interface to support motor imagery practice in people with stroke for upper limb recovery: a feasibility study. J Neuroeng Rehabil 2010, 7:60.

11. Pfurtscheller G, Neuper C, Flotzinger D, Pregenzer M: EEG-based discrimination between imagination of right and left hand movement. Electroencephalogr Clin Neurophysiol 1997, 103:642-651.

12. Qin $\mathrm{L}, \mathrm{He} B$ : A wavelet-based time-frequency analysis approach for classification of motor imagery for brain-computer interface applications. J Neural Eng 2005, 2(4):65.

13. Yuan H, Doud A, Gururajan A, He B: Cortical imaging of event-related (de)synchronization during online control of brain-computer interface using minimum-norm estimates in frequency domain. IEEE Trans Neural Syst Rehabil Eng 2008, 16:425-431.

14. Herman P, Prasad G, McGinnity T, Coyle D: Comparative analysis of spectral approaches to feature extraction for EEG-based motor imagery classification. IEEE Trans Neural Syst Rehabil Eng 2008, 16(4):317-326.

15. Krusienski DJ, McFarland DJ, Wolpaw JR: Value of amplitude, phase, and coherence features for a sensorimotor rhythm-based brain computer interface. Brain Res Bull 2012, 87:130- 134

16. McFarland DJ, Sarnacki WA, Wolpaw JR: Electroencephalographic (EEG) control of three-dimensional movement. J Neural Eng 2010, 7(3):036007

17. Do AH, Wang PT, King CE, Abiri A, Nenadic Z: Brain-computer interface controlled functional electrical stimulation system for ankle movement. J Neuroeng Rehabil 2011, 8:49.

18. Veluvolu KC, Wang Y, Kavuri SS: Adaptive estimation of EEG-rhythms for optimal band identification in BCI. J Neurosci Methods 2012, 203:163-172.

19. McFarland DJ, Wolpaw JR: Sensorimotor rhythm-based brain computer interface $(\mathrm{BCI})$ : model order selection for autoregressive spectral analysis. J Neural Eng 2008, 5:155-162.

20. Pfurtscheller G, Neuper C, Schlogl A, Lugger K: Separability of EEG signals recorded during right and left motor imagery using adaptive autoregressive parameters. IEEE Trans Neural Syst Rehabil Eng 1998, 6(NO.3):316-325.

21. Kiymik MK, Guler I, Dizibuyuk A, Akin M: Comparison of STFT and wavelet transform methods in determining epileptic seizure activity in EEG signals for real-time application. Comput Biol Med 2005, 35:603-616.

22. Allen DP, Mackinnon CD: Time frequency analysis of movementrelated spectral power in EEG during repetitive movements: $A$ comparison of methods. J Neurosci Methods 2010, 186:107-115.

23. Veluvolu KC, Ang WT: Estimation and filtering of physiological tremor for real-time compensation in surgical robotics applications. Int J Med Robot Comput Assist Surg 2010, 6:334-342.

24. Latt WT, Veluvolu KC, Ang WT: Drift-free position estimation of periodic or quasi-periodic motion using inertial sensors. Sensors 2011, 11(6):5931-5951.

25. Gabor D: Theory of communication. 26. Electrical Eng - Part III:Radio Commun Eng J Inst 1946, 93:442-445.

26. Mallat S: a Wavelet Tour of Signal Processing. Burlington: Elsevier; 2009.
27. Daubechies I: The wavelet transform, time-frequency localization and signal analysis. IEEE Trans Inf Theory 1990, 36(5):961-1005.

28. Zhan Y, Halliday D, Jiang P, Liu X, Feng J: Detecting time-dependent coherence between non-stationary electrophysiological signals- $A$ combined statistical and time-frequency approach. $J$ Neurosci Methods 2006, 156:322-332

29. Goupillaud P, Grossmann A, Morlet J: Cycle-octave and related transforms in seismic signal analysis. Geoexploration 1984, 23:85-102.

30. Aguiar-Conraia L, Soares MJ: The continuous wavelet transform: a primer. NIPE Working Paper Series 2011, NIPE WP 16:1-46.

31. Vaz CA, Thakor NV: Adaptive Fourier estimation of time-varying evoked potentials. IEEE Trans Biomed Eng 1989, 36(4):448-455.

32. Wang Y, Veluvolu KC, Cho JH, Defoort M: Adaptive estimation of EEG for subject-specific reactive band identification and improved ERD detection. Neurosci Lett 2012, 528(2):137-42.

33. Haykin S: Adaptive Filter Theory. Upper Saddle River: Prentice Hall; 2002.

34. Tarvainen MP, Hiltunen JK, Ranta-aho PO, Karjalainen PA: Estimation of nonstationary EEG with Kalman smoother approach: an application to event-related synchronization (ERS). IEEE Trans Biomed Eng 2004 51(3):516-524.

35. Shumway RH, Stoffer DS: Time Series Analysis and Its Applications. New York: Springer; 2000

36. Simon D: Optimal State Estimation. New Jersey: Wiley; 2006.

37. Guo L: Estimating time-varying parameters by the Kalman filter based algorithm: stability and convergence. IEEE Trans Automatic Control 1990, 35(2):141-147.

38. Guo L, Ljung L: Exponential stability of general tracking algorithms. IEEE Trans Automatic Control 1995, 40(8):1376-1387.

39. Ljung L, Gunnarsson S: Adaptation and tracking in system identification: a survey. Automatica 1990, 26:7-21.

40. Cao L, Schwartz HM: Exponential convergence of the Kalman filter based parameter estimation algorithm. Int J Adaptive Control Signal Process 2003, 17(10):763-783.

41. Brunner C, Naeem M, Leeb R, Graimann B, Pfurtscheller G: Spatial filtering and selection of optimized components in four class motor imagery data using independent components analysis. Pattern Recognit Lett 2007, 28:957-964.

42. Graimanna B, Huggins J, Levineb S, Pfurtscheller G: Visualization of significant ERD/ERS patterns in multichannel EEG and ECoG data. Clin Neurophysiol 2002, 113:43-47.

43. Chang DC, Wu WR: Maneuvering target tracking with high-order correlated noise - a multirate Kalman filtering approach. Wireless Pers Commun 2001, 17:103-123.

44. Rockmore D: The FFT: an algorithm the whole family can use. Comput SciEng 2000, 2:60-64.

45. Do AH, Wang PT, King CE, Abiri A, Nenadic Z: Brain computer interface controlled functional electrical stimulation system for ankle movement. J NeuroEng Rehabil 2011, 8:1-21.

doi:10.1186/1743-0003-10-109

Cite this article as: Wang et al:: Time-frequency analysis of band-limited EEG with BMFLC and Kalman filter for BCl applications. Journal of NeuroEngineering and Rehabilitation 2013 10:109. 